\title{
Pathophysiology of Autoimmune Thrombocytopenia: Current Insight with a Focus on Thrombopoiesis
}

\author{
Irene Marini ${ }^{1}$ Tamam Bakchoul ${ }^{1}$ \\ ${ }^{1}$ Department of Transfusion Medicine, Medical Faculty of Tübingen, \\ University of Tübingen, Tübingen, Germany \\ Hämostaseologie 2019;39:227-237.
}

\begin{abstract}
Address for correspondence Tamam Bakchoul, MD, Department of Transfusion Medicine, Medical Faculty of Tübingen, University Hospital of Tübingen, Otfried-Müller-Straße 4/1, 72076 Tübingen, Germany (e-mail: tamam.bakchoul@med.uni-tuebingen.de).
\end{abstract}

\begin{abstract}
Keywords

- autoantibodies

- platelet

- megakaryocytes

- autoimmune

disorders

- acquired thrombocytopenia
\end{abstract}

Immune thrombocytopenia (ITP) is an autoimmune disease characterized by a significant reduction in the number of circulating platelets which is frequently associated with bleeding. The total count of platelets in the body is finely regulated by the balance between platelet production and destruction. Although the pathogenesis of ITP is still not completely elucidated, it is largely recognized that the low platelet count observed in ITP patients is due to alterations of both mechanisms. An abnormal proliferation of autoreactive $T$ cells leading to the breakdown of immune tolerance to platelet antigens is suggested to be responsible for the up-regulated proliferation of autoantibody producing $B$ cells. Consequently, the immune response induces enhanced T cell-mediated cytotoxicity and antibody-mediated platelet destruction through phagocytosis, complement activation and apoptosis. An additional contribution to the pathophysiology of ITP is given by alterations of thrombopoiesis caused by platelet-reactive autoantibodies or cytotoxic $T$ cells leading to impaired megakaryocyte differentiation and platelet production. All these processes involved in ITP pathophysiology account for the complexity and heterogeneity in the clinical manifestation and therapy responsiveness of this disorder. For this reason, a better understanding of the different underlying mechanisms in ITP is necessary to develop more efficient therapeutic treatments in the future. In this review, we will provide an update on the pathophysiology of ITP with a particular focus on the impact of impaired thrombopoiesis.

\section{Introduction}

Immune thrombocytopenia (ITP) is an autoimmune bleeding disorder characterized by bleeding due to isolated thrombocytopenia with platelet count less than $100 \times 10^{9} / \mathrm{L}^{1-3} \mathrm{The}$ incidence of ITP ranges between 3.3 and 3.9 per 100,000 per year in adults, and between 1.9 and 6.4 per 100,000 per year in children. ${ }^{3,4}$ While a brief course with spontaneous remission is frequently observed in the majority of children with ITP, most adult patients display chronic ITP which can be associated with clinically significant bleeding including haemorrhages in skin or mucous membranes such as petechiae, purpura and rarely intracranial manifestations. ${ }^{5,6}$ Based on

received

July 19, 2018

accepted after revision

January 8, 2019

these clinical symptoms the primary therapeutic aim in ITP is to reduce the risk of severe bleeding.

The exact mechanism of autoimmunity leading to ITP is still unclear, but includes an alteration of the balance between effectors and regulatory cells. ${ }^{7}$ This imbalance results in a breakdown of the immune tolerance causing increased platelet clearance and impaired thrombopoiesis. Similarly to other autoimmune disorders, molecular mimicry with bacterial or viral proteins might be one reason for pathogenesis of ITP. In fact, it was reported a cross-reactivity of anti-platelet autoantibodies with human immunodeficiency virus, hepatitis $\mathrm{C}$ virus and Helicobacter pylori in secondary ITP., ${ }^{8,9}$ Under nonpathological conditions the immune system is finely regulated

(c) 2019 Georg Thieme Verlag KG Stuttgart · New York
DOI https://doi.org/ $10.1055 / \mathrm{s}-0039-1678732$. ISSN 0720-9355. 
by humoral and cellular components, including primarily regulatory $T$ and $B$ cells ( $T$ regs and $B$ regs). Functional alterations in these regulatory immune cells in ITP patients have been observed and are thought to contribute to ITP pathogenesis. For a long time, it was thought that the low platelet count is solely caused by enhanced destruction of platelets opsonized by antiplatelet antibodies. ${ }^{10-12}$ However, recent studies have shown that $\mathrm{T}$ cell cytotoxicity and impaired megakaryopoiesis are additional pathomechanisms in ITP. In this review, we will focus on the mechanisms leading to platelet destruction in ITP with a particular focus on current findings concerning alterations of thrombopoiesis.

\section{Pathophysiology of ITP}

\section{T Cell in ITP; Dysfunction in Regulation and Effector Function}

The loss of immunological tolerance to autoantigens expressed on patients' own platelets is one of the critical issues in the pathophysiology of ITP. In this context, several studies reported $\mathrm{T}$ cell abnormalities with an imbalance in $\mathrm{T}$ helper (Th)1/Th2 ratio in ITP patients. ${ }^{13,14} \mathrm{~T}$ regs are another subgroup of immune cells that play a critical role in maintaining self-tolerance under physiological conditions. These cells suppress cell- and antibody-mediated autoimmune responses. ${ }^{15}$ Dysfunction of these cells is thought to be involved in pathogenesis of ITP. ${ }^{16,17}$ For instance, $T$ regs were found to have reduced in vitro immunosuppressive activity. ${ }^{18}$ Moreover, an association was demonstrated between the severity of the disease (bleeding and thrombocytopenia) and the reduction of $\mathrm{T}$ regs. ${ }^{19}$ In fact, using a murine model, an increased severity of thrombocytopenia after depletion of CD8 $\mathrm{T}$ cell has been reported. While upon CD8 $\mathrm{T}$ regs re-transfusion correction in platelet counts was observed, indicating that $\mathrm{CD} 8+\mathrm{T}$ regs are required to maintain the immunological tolerance. ${ }^{20}$ These findings were supported by an additional study performed using an animal model. Nishimoto et al showed that approximately $40 \%$ of $\mathrm{T}$ reg-deficient mice developed thrombocytopenia which lasted for up to 5 weeks. Thrombocytopenic mice produced immunoglobulin $\mathrm{G}(\mathrm{IgG})$ anti-platelet antibodies which mainly target the glycoprotein (GP) Ib/IX. Of note, transfer of purified $\mathrm{T}$ regs into the deficient mice restored platelet count. ${ }^{21}$ Taking into consideration that up to $50 \%$ of ITP patients have no detectable autoantibodies in their blood, an alternative mechanism for platelet destruction in ITP is very obvious. $^{22,23}$ In fact, an increased number of $\mathrm{T}$ cells and higher cytotoxic activity have been reported in ITP patients with no detectable anti-platelet antibodies. ${ }^{24}$ In the same line, increased levels of splenic $T$ cells were detected in ITP patients who did not respond to the anti-B cell depleting treatment. These results support the hypothesis that $\mathrm{T}$ cells are involved in the pathophysiology of ITP in a B cellindependent manner. So far, the target peptides bound by platelet-specific $\mathrm{T}$ cells expressed on major histocompatibility complex (MHC) class I have not been identified. Interestingly, a study proposed that platelets preferentially activate naïve $T$ cells and that they can express the pathogen-derived peptides in the context of MHC class I. ${ }^{25}$ Although the role of antigen presentation on MHC class I in ITP development is still unclear, this represents a promising area for further investigations.

Accumulating evidence suggests that cytotoxic T lymphocytes contribute directly to the increased platelet destruction. ${ }^{24,26}$ It was shown that ITP patients have autoreactive T cell clones against cryptic GPIIb/IIla epitopes ${ }^{27}$ and enhancement of oligoclonal T cells. ${ }^{28}$ In addition, $\mathrm{T}$ cells from ITP patients were found to harbour increased cytotoxicity against autologous platelets. ${ }^{29}$ In active ITP patients, lysis of radiolabelled autologous platelets by purified CD3 + CD8 + T cells as effector cells was observed, but not in ITP patients in remission. ${ }^{26}$ Apoptosis has also been suggested as another mechanism of platelet destruction caused by activated autoreactive $T$ cells. This hypothesis is supported by the observation that purified CD8 + T cells from ITP patients overexpress molecules involved in cytotoxicity and induce upregulation of the apoptotic marker annexin $\mathrm{V}$ in autologous platelets. ${ }^{26}$ Based on these findings, the concept that apoptosis and perforin/granzyme-mediated cytotoxicity represent an important pathway through which cytotoxic $T$ cells destroy autologous platelets in ITP patients is corroborated.

\section{B Cell in ITP; Activated Humoral Autoimmune Response} It is generally accepted that ITP is associated with dysfunctions of $B$ cells. The contribution of $B$ cells to the pathogenesis of ITP is not restricted to the production of autoantibodies. Enhanced numbers of $B$ cells have been observed in the red pulp spleen sections of ITP patients, suggesting that these cells contribute to the autoantigen stimulation in ITP..$^{30,31}$ The development and survival of $B$ cells is regulated by the $B$ cell activating factor of the TNF family (BAFF). ${ }^{32}$ Several studies showed higher serum levels of BAFF in untreated ITP patients, which were significantly reduced upon successful immunosuppressive therapy. ${ }^{33}$ Moreover, the up-regulation of THR7 in mice with ITP led to an enhancement in BAFF, with a subsequent decrease in platelet count. ${ }^{34,35}$ These findings indicate that survival of autoreactive B cells is enhanced by BAFF in ITP patients.

Recently, several studies provided robust evidence concerning the involvement of $\mathrm{B}$ regs in chronic ITP patients. ${ }^{36,37} \mathrm{~B}$ regs (CD19 + CD24hiCD38hi) maintain peripheral tolerance by secretion of IL-10 leading to $T$ regs recruitment and/or differentiation, and reduction of $\mathrm{CD} 4+\mathrm{T}$ cell functionality. ${ }^{38,39}$ Notably, functional impairment of B regs was observed in non-splenectomized ITP patients with chronic ITP. ${ }^{36}$

The presence of autoantibodies produced by B cells in ITP is fully established. ${ }^{40}$ The first demonstration of the pathogenic effect of these antibodies took place in the early 1950s. Harrington and Hollingssworth demonstrated, for the first time, the existence of a 'thrombocytopenic factor' in the blood of ITP patients. They induced transient thrombocytopenia in healthy recipients who received $500 \mathrm{~mL}$ of whole blood from ITP patients. In these subjects a significant reduction in platelet count after 2 hours and a complete absence of platelets after 24 hours, with a gradual recovery after a few days, ${ }^{41}$ were observed. Years later, Shulman et al revealed that the 
'thrombocytopenic factor' was present in the IgG fraction of plasma leading to the hypothesis that the anti-platelet factor was an antibody. ${ }^{10}$ Finally, at the end of the 1990 s, it was found that the antibodies are mainly directed against platelet surface proteins. ${ }^{42,43}$ Based on these findings, intensive efforts have been taking place for more than two decades to identify the antigen specificity of antibodies in ITP. A deeper understanding was achieved with the introduction of immunocapture assays, in particular the monoclonal antibody-specific immobilization of platelet antigens (MAIPA). ${ }^{44}$ Using this assay, it has been demonstrated that autoantibodies in ITP are mainly directed against GPIIb/IIIa and GPIb/IX, but also GPIV, GPVI, GPIa/IIa and GPV. ${ }^{45}$ More recent research revealed that autoantibodies recognize several epitopes and require different conformations. Anti-GPIIb/IIIa antibodies, for example, frequently bind to cation-dependent conformational antigens on $\alpha \mathrm{IIb} \beta 3$, but not to another $\beta 3$-containing integrin, $\alpha v \beta 3 .{ }^{46,47}$ These autoantibodies bind specifically to the $\beta$-propeller domain of $\alpha$ IIb, predominantly from the N-terminus to the W4:4-1 loop of the $\beta$-propeller domain. Interestingly, it was observed that in the presence of a single amino acid substitution in $\alpha$ IIb, the reactivity of some autoantibodies is completely inhibited, suggesting that target epitopes are extremely restricted to conformational epitopes. ${ }^{48} \mathrm{~A}$ similar effect was observed by He et al for antibodies targeting the GPIb/IX, which mainly recognize a short amino acid sequence (333-341) on GPIb $\alpha .{ }^{43}$ Although most antibodies bind the GPIb component of the receptor complex GPIb/IX/V, little is known about relevant autoepitopes on the entire complex. ${ }^{49-51}$ Given the variety of the target antigens, we have summarized the characteristics of GPs targeted by ITP autoantibodies in -Table 1 .

Despite this heterogeneity in target antigens, anti-GPIIb/ IIIa antibodies have been shown to have restricted $\mathrm{k} / \lambda$-chain usage. ${ }^{48,52}$ Roark and colleagues demonstrated by sequencing analysis of the Fab region of immunoglobulin arrangements using phage display libraries constructed from splenocytes of ITP patients that anti-platelet autoantibodies in ITP use highly limited immunoglobulin variable regions. ${ }^{53}$ Moreover, they have noticed a selective incorporation of the VH3-30 variable heavy chain gene segment suggesting that the antigenic repertoire in ITP is fairly limited and that antiplatelet antibodies are generated from a restricted number of B cell clones.
Taken together, immune dysregulation in ITP is not limited to $\mathrm{T}$ cell subsets. B cells play a central role as a regulatory cell as well as effector cells in a complex molecular network that has not been elucidated so far.

\section{Mechanisms Leading to Autoantibody-Mediated Platelet Clearance}

Autoantibody-mediated thrombocytopenia can be induced by different mechanisms such as phagocytosis of platelets upon antibody binding, apoptosis, desialylation, complement activation and impairment of platelet production (-Fig. 1). Interestingly, in addition to the fast clearance of platelets, ITP-autoantibodies are able to induce functional alterations of the circulating cells. In fact, although it is extremely difficult to investigate platelet functions in subjects with low platelet counts, functional defects in patients characterized by severe bleeding have been reported. ${ }^{54}$ In particular, a decrease of platelet degranulation after ADP stimulation as well as a reduced ability to form microaggregates upon stimulation have been shown in ITP patients. In accordance with these findings, another group observed decreased levels of P-selectin- and activated GPIIb/IIIa-positive platelets upon adenosine diphosphate (ADP) or thrombin receptor-activating peptide (TRAP) activation in paediatric ITP patients with severe bleeding. ${ }^{55}$

\section{Fc-Dependent Platelet Phagocytosis}

Opsonized platelets are primarily destroyed by macrophages in the spleen. This process is mediated by low affinity Fc gamma receptors (FcgRs) IIA and FcgRIIIA which are linked to immune receptor tyrosine-based activation motifs (ITAMs) in the intracellular domain. Upon autoantibody binding, ITAMs are phosphorylated by the tyrosine kinase Syk, triggering the phagocytosis. ${ }^{56,57}$ Although all FcgRs are involved in activating the immune system, FcgRIIB is the only inhibitory Fc receptor. This is due to its immune receptor tyrosinebased inhibitory motif (ITIM) in the cytoplasmatic tail which inhibits phagocytosis and the release of pro-inflammatory cytokines by macrophages and dendritic cells. In this context, the ratio between different FcgRs is critical to correctly regulate the immune response. In fact, in patients responding to $H$. pylori eradication, a shift in monocyte FcgRs expression toward FcgRIIB has been reported. ${ }^{58}$ The relevant

Table 1 Characteristics of glycoproteins (GPs) targeted by ITP autoantibodies

\begin{tabular}{|l|l|l|l|l|}
\hline $\begin{array}{l}\text { Targets of } \\
\text { AAbs }\end{array}$ & Copies/PLT & Ligand & Functions & Related disorders \\
\hline $\begin{array}{l}\text { GPIIb/IIla } \\
\left(\alpha \mathrm{llb} \beta_{3}\right)\end{array}$ & $50,000-80,000$ & Fibrinogen & Platelet adhesion and aggregation & Glanzmann's syndrome \\
\hline GPIb/IX/V & $\sim 50,000$ & von Willebrand factor & Platelet adhesion and aggregation & Bernard-Soulier syndrome \\
\hline GPIV & $12,000-25,000$ & Collagen & Platelet activation and aggregation & - \\
\hline GPla/Ila & $2,000-4,000$ & Collagen & Platelet activation and aggregation & - \\
\hline$\alpha_{v} \beta_{3}$ & $300-400$ & Vitronectin & Platelet adhesion and aggregation & - \\
\hline
\end{tabular}

Abbreviations: AAbs, autoantibodies; ITP, immune thrombocytopenia; PLT, platelet.

Source: Clemetson KJ, Clemetson JM. Platelet receptors. In: Michelson AD, ed. Platelets. 3rd ed. San Diego, CA: Elsevier/Academic Press; $2013 ; 169-194$. 


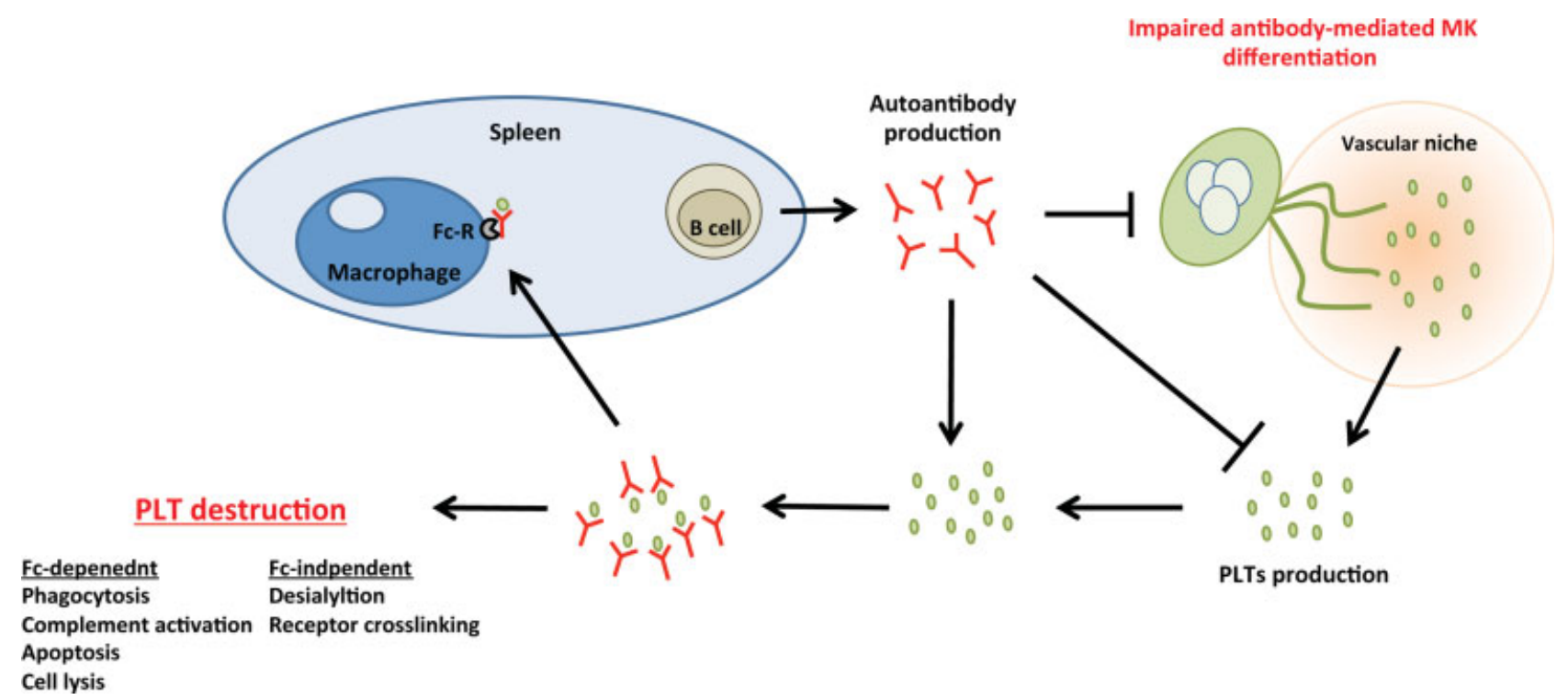

Fig. 1 Mechanisms causing platelet destruction in immune thrombocytopenia. Platelets (PLTs) are destroyed in the circulation through different mechanisms. Autoantibodies, produced by B cells, can induce phagocytosis, complement activation, apoptosis and cell lysis via their $\mathrm{Fc}$ domain. In addition, Fc-independent platelet destruction mechanisms have been suggested recently including desialylation and activation. (Adapted from Kashiwagi et al 2013, International Journal of Hematology.)

role of the FcgRIIB was also demonstrated in a study, using animal model, which showed that its presence on splenic macrophages is necessary for the protective effects of intravenous immunoglobulin (IVIG). ${ }^{59}$

\section{Complement Activation}

Complement activation represents an additional important mechanism of platelet destruction. Several in vitro studies ${ }^{60,61}$ documented antibody-mediated complement fixation to platelets from ITP patients. GPIIb/IIIa and GPIb/IX are the major targets of autoantibodies with complement fixation capability. ${ }^{62}$ The biological significance of this observation was demonstrated using an established mouse model of ITP. ${ }^{63}$ The authors showed that platelet destruction by complement-fixing autoantibodies is significantly increased in the presence of complement. ${ }^{64}$ Taking these findings into account, novel therapies for ITP could target complement factors. For instance, eculizumab was shown to rescue thrombocytopenia in patients with anti-phospholipid syndrome by inhibition of the downstream components of complement.

\section{Fc-Independent Platelet Destruction}

The Fc-mediated platelet phagocytosis is a well-known mechanism in ITP pathogenesis. However, it has been estimated that $20 \%$ of ITP patients are refractory to the standard therapies that target the Fc receptor signalling, ${ }^{65}$ including splenectomy and IVIG. This indicates that, at least for this subgroup of patients, the antibody-mediated platelet destruction involves different pathways and site of clearance. Recent studies proposed two Fc-independent mechanisms. ${ }^{66,67}$ Using a murine model, Li and coworkers showed that ITP autoantibodies induce glycan modification of platelet surface GPs, which are recognized by Ashwell-Morell receptors, expressed on hepatocytes, leading to accelerated platelet clearance in the liver. ${ }^{68}$ In some patients, this may explain the ineffectiveness of splenectomy which represents the ultimate ITP therapeutic option for refractory subjects. Interestingly, 2 years later a retrospective study involving 61 ITP patients reported a correlation between platelets desialylation and a reduced response to first-line treatments corroborating the hypothesized Fc-independent mechanism. ${ }^{69,70}$ Another Fc-independent mechanism has been suggested by Quach et al who showed that non-responding ITP patients often produce autoantibodies targeting the ligand binding domain of GPIb/IX. This specific binding can activate GPIb/IX by platelet receptorcrosslinking, inducing unfolding of its mechanosensory domain and the consequent platelet destruction. ${ }^{71}$ However, it is currently unclear whether the unfolding of GPIb mechanosensory domain is the earlier event required for sialidase neuraminidase-1 translocation and platelet desialylation.

\section{Antibody-Mediated Platelet Apoptosis}

The platelet life cycle is regulated by the intrinsic apoptotic pathway similarly to nucleated cells. Considering this, the contribution of ITP autoantibodies in inducing platelet apoptosis was investigated by several groups using well-defined apoptosis markers such as depolarization of the mitochondrial transmembrane potential, Bcl-2 family protein expression, caspase- 3 and 9 activation and phosphatidylserine (PS) exposure. ${ }^{72-74}$ One of the first studies performed using a mouse model for ITP showed that upon injection of anti-GPIIb antibodies, murine platelets displayed apoptotic features including destruction of the mitochondrial potential, caspase activation and PS expression. ${ }^{75}$ In human ITP, platelet apoptosis has been reported in paediatric and adult patients with acute ITP which was ameliorated by immunoglobulin infusion. ${ }^{76,77}$ Interestingly, in a recent study apoptotic platelets were found in ITP patients expressing antiGPIIb/IIIa and anti-GPIb autoantibodies but not in those carrying anti-GPIa/Ila autoantibodies. ${ }^{78}$ This suggests a possible preferential specificity of the autoantibodies in inducing platelets 
apoptosis. Although, the exact mechanism of autoantibodymediated platelet apoptosis is not yet completely clear, these findings suggest a relevant contribution of the apoptotic pathway in the ITP pathogenesis, opening novel horizons for deeper investigations.

\section{Physiological Thrombopoiesis}

In addition to the established mechanisms leading to platelet destruction, an equivalent role in the pathophysiology of ITP is played by antibody-mediated impaired platelet production. Megakaryopoiesis is a complex process that takes place in the bone marrow involving molecular and cellular changes leading to biogenesis of platelets. The primary activator and regulator of the process is the thrombopoietin (TPO) produced in the liver. ${ }^{79,80}$ TPO triggers the differentiation of haematopoietic stem cells (HSCs) into polyploid cells, known as megakaryocytes. Upon complete maturation, megakaryocytes develop proplatelet extensions through the endothelial cells of the vascular niche and finally release platelets into the blood stream (almost 10,000 platelets per megakaryo- cyte and a total of 100 million each day in healthy subjects). ${ }^{81}$ Bone marrow-derived mesenchymal stem cells (MSCs) are essential components in the haematopoietic microenvironment and provide support to megakaryopoiesis. They secrete a wide range of cytokines including interleukin 6 (IL-6), IL10, IL-11, prostaglandins, stem cell factor (SCF) and leukaemia inhibitory factor which induce megakaryocyte biogenesis and maturation. ${ }^{82}$ These cells are capable of self-renewal and differentiation by which they maintain the correct balance between HSC and megakaryocyte differentiation in the bone marrow. Clearly, alterations in any stage of the megakaryopoiesis will affect platelet production ( - Fig. 2).

\section{Thrombopoiesis in ITP}

The first observations of morphological alterations on megakaryocytes from ITP patients were reported in the 1940s. An increase of immature megakaryocytes, characterized by abnormalities in the ploidy state of the nucleus and cytoplasm, was observed in the bone marrow of ITP patients using light microscopy ${ }^{83}$ Interestingly, after transfusion of ITP serum into

\section{Normal thrombopoiesis}

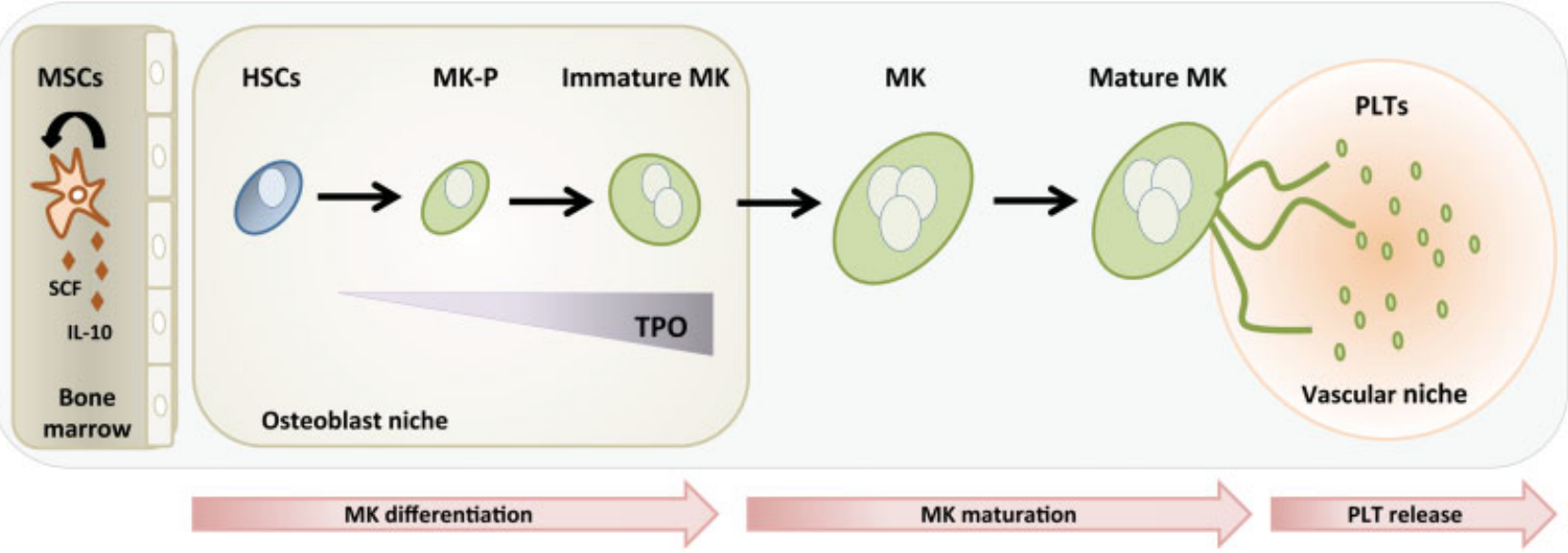

Thrombopoiesis in ITP patients

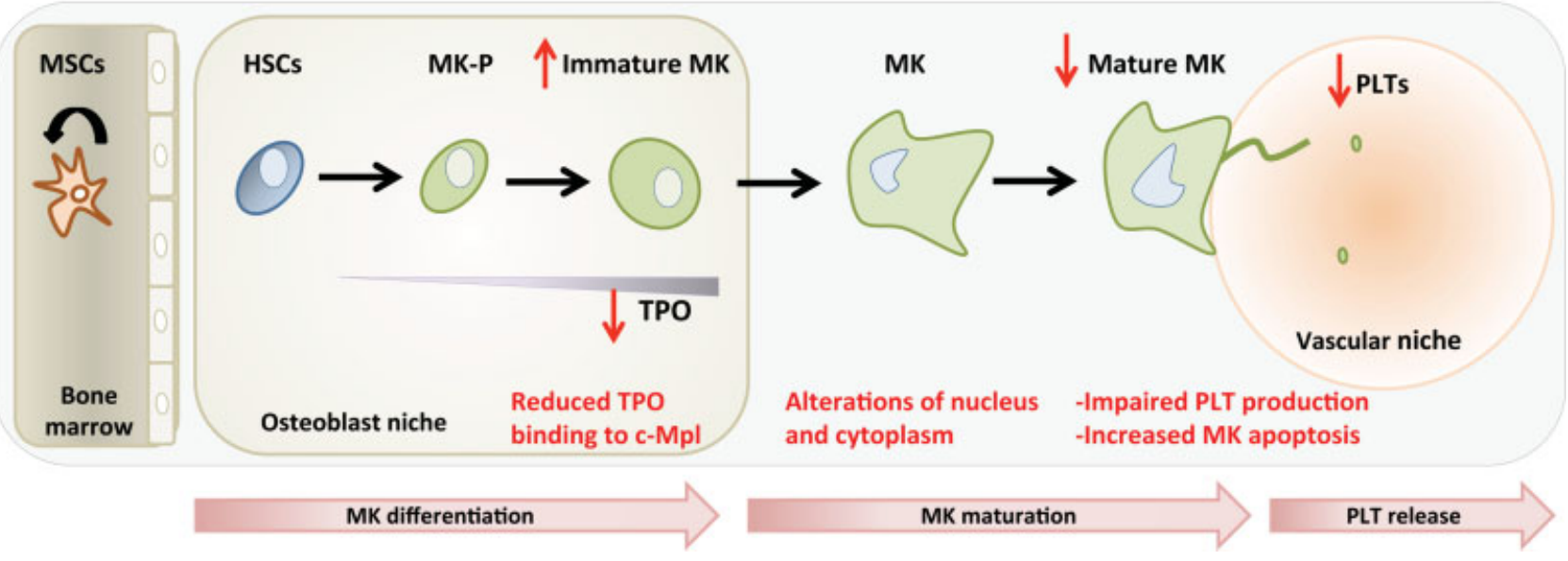

Fig. 2 Altered thrombopoiesis in immune thrombocytopenia (ITP) patients. Upper panel: in physiological condition, thrombopoietin (TPO) induces the differentiation of haematopoietic stem cells (HSCs) into megakaryocyte progenitors (MK-P), immature MKs and finally into the mature MKs characterized by polyploid nucleus. These cells develop proplatelet extensions and release platelets (PLTs) in the blood stream. Mesenchymal stem cells (MSCs), residing in the bone marrow, support the entire process maintaining the balance between self-renewal and differentiation. Lower panel: during thrombopoiesis in ITP patients, different steps of the entire process are altered as shown in the figure (Adapted from Eto K. and Kunishima S., 2016, Blood.) 
healthy subjects, the same alterations on megakaryocyte morphology were detected. ${ }^{84}$ This finding can be explained by the fact that GPIb and GPIIb/IIIa, the major targets of ITP autoantibodies, are expressed not only on the surface of platelets but also on megakaryocytes during their differentiation. The autoantibody binding results in suppression of megakaryocyte maturation and platelet formation. ${ }^{85,86}$ In fact, the antibodymediated inhibition of platelet production was demonstrated by in vitro studies using HSCs that differentiated into megakaryocytes in the presence of ITP autoantibodies targeting GPIb/IX and GPIIb/IIIa. In those studies, impaired megakaryocyte maturation and decreased platelet formation were observed. ${ }^{87-89}$ In a more recent study, Zeng et $\mathrm{al}^{90}$ have investigated the impact of anti- $\alpha v \beta 3$ autoantibody, which is expressed in chronic ITP patients, on megakaryocyte differentiation, survival, migratory and adhesive ability. In fact, the migration and adhesion of megakaryocytes in the vascular niche is an essential process for an efficient thrombopoiesis in the human body. Notably, impaired migration and adhesion of megakaryocytes, but a normal viability and proliferation of the cells, have been observed in the presence of anti- $\alpha \mathrm{v} \beta 3$ autoantibody. These findings suggest that anti- $\alpha v \beta 3$ autoantibodies might have a selective inhibitory impact on megakaryocyte adhesion and migration during ITP pathogenesis. Furthermore, using a murine model the authors reported a lower count of megakaryocytes residing in the vascular niche in ITP mice. Interestingly, bone marrow biopsies of ITP patients, which express anti- $\alpha \mathrm{v} \beta 3$ antibody, have shown similar phenotype, corroborating the in vitro and in vivo findings. The results of this study indicate that anti- $\alpha v \beta 3$ autoantibody might reduce megakaryocyte differentiation and platelet production through inhibition of the megakaryocyte's migration ability.

Whereas conventional therapeutic approaches in ITP aimed mainly to reduce immune-mediated platelet destruction, newer treatments seek to increase thrombopoiesis and the consequent platelet production. Megakaryopoiesis suppression has been observed in ITP patients with autoantibodies targeting specifically the TPO receptor (TPO-R) c-Mpl. ${ }^{91}$ As a key activator and regulator of the platelet production process, the activation of $\mathrm{c}-\mathrm{Mpl}$ receptor was one of the successful therapeutic targets investigated. In fact, platelet count increases have been addressed using TPO-R agonists such as romiplostim and eltrombopag. ${ }^{92,93}$ Clinical confirmation of the efficacy of these substances emerged from several randomized trials, performed during long-term treatment, which reported a 70 to $80 \%$ response in refractory ITP patients. ${ }^{94,95}$ Recently, Bal et al reported an additional therapeutic effect of TPO-R agonists showing increased proliferation, mobilization and differentiation of HSCs and early cell progenitors of all three haematopoietic lineages ${ }^{96}$ Consequently, HSCs seem to be a promising tool for novel cell-based therapies in ITP.

Genetic factors with a potential association to ITP have been investigated extensively; however, it is still difficult to define a clear conclusion from the data currently available. Nevertheless, it has been reported that microRNAs (miRNAs), small non-coding RNA molecules that regulate gene expression by targeting messenger RNA (mRNA), ${ }^{97}$ change under pathological conditions. Interestingly, although the role of mRNA in ITP has not been completely elucidated, ${ }^{98,99}$ several mRNAs seem to have an impact on megakaryocyte differentiation. For instance, $\beta-1$ tubulin $\mathrm{R} 307 \mathrm{H}$ singlenucleotide polymorphism (SNP) was suggested to be a potential biomarker in ITP. It has been shown that 30\% of ITP patients present SNP allele, and any difference observed in relation to SNP might influence a large number of patients. The relationship between changes in platelet physiology and changes in isotype of tubulin (as the main component of marginal band platelet) highlights the possible role of $\beta-1$ tubulin in platelet activity. ${ }^{100,101}$

\section{Megakaryocyte Apoptosis}

An interesting open question is the role of megakaryocyte apoptosis in the ITP pathophysiology. Controversial results were presented in several studies during the last few years. In fact, it has been reported that ITP plasma can reduce megakaryocyte apoptosis. ${ }^{102}$ In particular, after cultivation of HSCs from healthy umbilical cord blood with ITP plasma, a decreased percentage of apoptotic cells, reduced expression of tumor necrosis factor-related apoptosis inducing ligand (TRAIL) and increased expression of the anti-apoptotic protein Bcl-xL have been observed in the differentiated megakaryocytes. ${ }^{103}$ In contrast, an in vivo study by Houwerzijl et al showed that megakaryocytes undergo apoptosis in the presence of autoantibodies displaying nuclear fragmentation, chromatin condensation and activation of caspase 3 in biopsies of ITP patients, leading to phagocytosis of the polyploid cells by macrophages residing in the bone marrow. ${ }^{104}$

Although the mechanisms of impaired megakaryopoiesis are not yet completely clear, it is well known that in bone marrow of ITP patients the number of megakaryocytes is normal or increased despite platelet production and count being reduced. ${ }^{105}$ In this context, the apoptotic pathways in megakaryocytes might represent an explanation. In fact, reduced apoptosis in megakaryocytes and their consequent long survival in the bone marrow without an effective megakaryopoiesis is a reasonable hypothesis. This theory is corroborated by a more recent study where megakaryocyte apoptosis was investigated in bone marrow samples of ITP patients. ${ }^{106}$ Vrbensky and coworkers have observed a decrease of megakaryocyte apoptosis in bone marrow biopsies of ITP subjects in comparison to healthy controls. In the same line, data from animal study showed that within 24 hours upon administration of different autoantibodies (anti- $\alpha$ IIb, anti- $\beta 3$ or anti-GPIb) all of them were able to develop thrombocytopenia in recipient mice. However, only two antibodies (anti$\alpha I I b$ and anti- $\beta 3$ ) have induced an alteration of the number of megakaryocytes in the bone marrow. This suggests that not all autoantibodies affect the megakaryocyte count and consequently a different mechanism may be the cause of megakaryocyte alterations during ITP. ${ }^{107}$

\section{Defective Mesenchymal Stem Cells in ITP}

Emerging studies are suggesting a contribution of the defective MSCs in the mechanism leading to ITP pathogenesis. One of the most important properties of these cells is their immunosuppressive function, involving both adaptive and 
innate immune responses. ${ }^{108}$ In fact, it was reported that MSCs could induce an immunosuppressive or tolerant phenotype in physiological conditions by altering the cytokine's secretion functions of dendritic cells, effector T cells (Th1 and Th2) and natural killer cells. ${ }^{109,110}$ In contrast, functional impairment of MSCs was observed in different autoimmune disorders including rheumatoid arthritis, ${ }^{111}$ systemic lupus erythematosus ${ }^{112}$ and aplastic anaemia. ${ }^{113}$ Accordingly with these observations, several investigations have demonstrated that MSCs from ITP patients are characterized by increased number of apoptotic cells and reduced capacity to inhibit the proliferation of activated T cells. ${ }^{14,115}$ Given these findings, defective MSCs are currently being explored as a potential target in the treatment of ITP. A few years ago it was reported that platelet-derived growth factor (PDGF-BB) has a protective effect on MSCs. PDGF-BB was found to act against apoptosis, senescence and immunomodulatory dysfunctions. These observations were corroborated by Tao et al who demonstrated that transplantation of healthy MSCs can rescue the functional immune phenotype in a murine model of ITP. The administration of MSCs induced a significantly decreased level of $\mathrm{T}$ regs and an increase in platelet count in ITP mice. ${ }^{116}$ These observations were further supported by a recent clinical study exhibiting a similar experimental setting that involved four patients with chronic refractory ITP. A complete remission was achieved in three patients within 12 months and for one patient after 24 months, without additional immunosuppressive drugs. Interestingly, during follow-up analysis no severe side effects were observed, suggesting that MSC transplantation seems to be a safe and efficient cell-based therapeutic approach to treat refractory ITP. ${ }^{117}$

\section{Conclusion}

ITP is a complex and multifactorial disease. In fact, the clinical manifestations as well as patients' response to different treatments are very heterogeneous suggesting that ITP is a group of disorders sharing common characteristics, namely loss of immune tolerance toward platelet (and megakaryocyte) antigens and dysfunctional primary haemostasis. Recent studies provided significant insight into the pathophysiology of ITP including identification of the role of antigen-presenting cells, T and B cells, and their interactions. Of most importance was the discovery that the abnormal functions of $\mathrm{B}$ regs and $\mathrm{T}$ regs, which are supposed to maintain self-tolerance, are involved in modulating ITP pathogenesis. The immunological consequence of the activated $B$ and $T$ cell-mediated immune response is the proliferation of platelet-specific plasma and cytotoxic cells, respectively. While the latter are responsible for a direct destruction of platelet as well as megakaryocyte in ITP, IgG autoantibodies generally directed at the most abundant platelet surface GPs (GPIIb/IIIa and GPIb/IX/V) can induce thrombocytopenia in ITP by several Fc-domain dependent mechanisms including platelet phagocytosis, complement activation, apoptosis, cell lysis and inhibition of proplatelet production. Additionally, recent data suggest that surface expression of sialic acid can be modified by autoantibodies leading to platelet clearance via Ashwell-Morell receptor (AMR) system in the liver in an Fc-independent manner. Of note, some patients show a decrease in autoantibody concentration after treatment with steroids but no significant improvement in platelet count (personal observation). Since only platelets are used in antibody tests, it could be possible that autoantibodies with higher avidity to megakaryocytes are still circulating but not detectable using standard assays. These autoantibodies with a preferential binding to megakaryocytes could induce thrombocytopenia via inhibition of thrombopoiesis. Although this hypothesis is speculative, future investigations could help understand the heterogeneity that is frequently observed in the responsiveness of ITP patients to different treatments. Future studies should also aim to investigate the increased platelet mass due to various therapies, and its role in presenting platelet autoantigens to $T$ cells and potential effects on activating and suppressing $\mathrm{T}$ regs, which may elucidate novel pathomechanisms. Taking the heterogeneity of ITP into consideration, improving our knowledge of the pathogenesis of ITP is an essential cornerstone for identifying effective diagnostic tests and efficient therapeutic approaches for this clinically relevant form of acquired thrombocytopenia.

\section{Author Contributions}

Irene Marini did the literature search and wrote the sections on alterations of megakaryopoiesis and thrombopoiesis. Tamam Bakchoul contributed to the subsections on the antibody-mediated platelet clearance. Both authors approved the final version of the manuscript.

\section{Conflict of Interest}

I.M. has no conflict of interests.

T.B. reports receiving honoraria for a scientific talk from Aspen Germany, CSL Behring and Stago gGmbH German, and research grants from the German Society of Research, the German Society for Transfusion Medicine and German Red Cross.

\section{Acknowledgment}

The authors thank Stephen Bosher for editing the manuscript as a native English speaker.

\section{References}

1 Provan D, Stasi R, Newland AC, et al. International consensus report on the investigation and management of primary immune thrombocytopenia. Blood 2010;115(02):168-186

2 Neunert C, Lim W, Crowther M, Cohen A, Solberg L Jr, Crowther MA; American Society of Hematology. The American Society of Hematology 2011 evidence-based practice guideline for immune thrombocytopenia. Blood 2011;117(16):4190-4207

3 Moulis G, Palmaro A, Montastruc JL, Godeau B, Lapeyre-Mestre M, Sailler L. Epidemiology of incident immune thrombocytopenia: a nationwide population-based study in France. Blood 2014; 124(22):3308-3315

4 Bennett CM, Neunert C, Grace RF, et al. Predictors of remission in children with newly diagnosed immune thrombocytopenia: 
data from the Intercontinental Cooperative ITP Study Group Registry II participants. Pediatr Blood Cancer 2018;65(1)

5 Cines DB, Liebman HA. The immune thrombocytopenia syndrome: a disorder of diverse pathogenesis and clinical presentation. Hematol Oncol Clin North Am 2009;23(06):1155-1161

6 D'Orazio JA, Neely J, Farhoudi N. ITP in children: pathophysiology and current treatment approaches. J Pediatr Hematol Oncol 2013;35(01):1-13

7 McKenzie CG, Guo L, Freedman J, Semple JW. Cellular immune dysfunction in immune thrombocytopenia (ITP). Br J Haematol 2013;163(01):10-23

8 Takahashi T, Yujiri T, Tanizawa Y. Helicobacter pylori and chronic ITP: the discrepancy in the clinical responses to eradication therapy might be due to differences in the bacterial strains. Blood 2004;104(02):594

9 Rajan SK, Espina BM, Liebman HA. Hepatitis C virus-related thrombocytopenia: clinical and laboratory characteristics compared with chronic immune thrombocytopenic purpura. Br J Haematol 2005;129(06):818-824

10 Shulman NR, Marder VJ, Weinrach RS. Similarities between known antiplatelet antibodies and the factor responsible for thrombocytopenia in idiopathic purpura. Physiologic, serologic and isotopic studies. Ann N Y Acad Sci 1965;124(02):499-542

11 Ku FC, Tsai CR, Der Wang J, Wang CH, Chang TK, Hwang WL. Stromal-derived factor-1 gene variations in pediatric patients with primary immune thrombocytopenia. Eur J Haematol 2013; 90(01):25-30

12 Rank A, Weigert O, Ostermann H. Management of chronic immune thrombocytopenic purpura: targeting insufficient megakaryopoiesis as a novel therapeutic principle. Biologics 2010;4:139-145

13 Zhao Z, Yang L, Yang G, et al. Contributions of T lymphocyte abnormalities to therapeutic outcomes in newly diagnosed patients with immune thrombocytopenia. PLoS One 2015;10 (05): 0126601

14 Ji X, Zhang L, Peng J, Hou M. T cell immune abnormalities in immune thrombocytopenia. J Hematol Oncol 2014;7:72

15 Sakaguchi S. Naturally arising Foxp3-expressing CD25+CD4+ regulatory $\mathrm{T}$ cells in immunological tolerance to self and nonself. Nat Immunol 2005;6(04):345-352

16 Sakakura M, Wada H, Tawara I, et al. Reduced Cd4+Cd25+ T cells in patients with idiopathic thrombocytopenic purpura. Thromb Res 2007;120(02):187-193

17 Liu B, Zhao H, Poon MC, et al. Abnormality of CD4(+)CD25(+) regulatory $\mathrm{T}$ cells in idiopathic thrombocytopenic purpura. Eur $\mathrm{J}$ Haematol 2007;78(02):139-143

18 Hemdan NY, Birkenmeier G, Wichmann G, et al. Interleukin-17producing T helper cells in autoimmunity. Autoimmun Rev 2010; 9(11):785-792

19 Ma D, Zhu X, Zhao P, et al. Profile of Th17 cytokines (IL-17, TGFbeta, IL-6) and Th1 cytokine (IFN-gamma) in patients with immune thrombocytopenic purpura. Ann Hematol 2008;87 (11):899-904

20 Ma L, Simpson E, Li J, et al. CD8 + T cells are predominantly protective and required for effective steroid therapy in murine models of immune thrombocytopenia. Blood 2015;126(02): 247-256

21 NishimotoT, Satoh T, Simpson EK, Ni H, Kuwana M. Predominant autoantibody response to GPIb/IX in a regulatory T-cell-deficient mouse model for immune thrombocytopenia. J Thromb Haemost 2013;11(02):369-372

22 Veneri D, De Matteis G, Solero P, et al. Analysis of B- and T-cell clonality and HLA class II alleles in patients with idiopathic thrombocytopenic purpura: correlation with Helicobacter pylori infection and response to eradication treatment. Platelets 2005; 16(05):307-311

$23 \mathrm{Kim} \mathrm{J}$, Park CJ, Chi HS, et al. Idiopathic thrombocytopenic purpura: better therapeutic responses of patients with B- or
T-cell clonality than patients without clonality. Int J Hematol 2003;78(05):461-466

24 Zhao C, Li X, Zhang F, Wang L, Peng J, Hou M. Increased cytotoxic T-lymphocyte-mediated cytotoxicity predominant in patients with idiopathic thrombocytopenic purpura without platelet autoantibodies. Haematologica 2008;93(09):1428-1430

25 Chapman LM, Aggrey AA, Field DJ, et al. Platelets present antigen in the context of MHC class I. J Immunol 2012;189(02):916-923

26 Zhang F, Chu X, Wang L, et al. Cell-mediated lysis of autologous platelets in chronic idiopathic thrombocytopenic purpura. Eur J Haematol 2006;76(05):427-431

27 Kuwana M, Kaburaki J, Ikeda Y. Autoreactive T cells to platelet GPIIb-IIla in immune thrombocytopenic purpura. Role in production of anti-platelet autoantibody. J Clin Invest 1998;102 (07):1393-1402

28 Stasi R, Del Poeta G, Stipa E, et al. Response to B-cell depleting therapy with rituximab reverts the abnormalities of T-cell subsets in patients with idiopathic thrombocytopenic purpura. Blood 2007;110(08):2924-2930

29 Olsson B, Andersson PO, Jernås M, et al. T-cell-mediated cytotoxicity toward platelets in chronic idiopathic thrombocytopenic purpura. Nat Med 2003;9(09):1123-1124

30 Daridon C, Loddenkemper C, Spieckermann S, et al. Splenic proliferative lymphoid nodules distinct from germinal centers are sites of autoantigen stimulation in immune thrombocytopenia. Blood 2012;120(25):5021-5031

31 Olsson B, Ridell B, Jernås M, Wadenvik H. Increased number of Bcells in the red pulp of the spleen in ITP. Ann Hematol 2012;91 (02):271-277

32 Youinou P, Pers JO. The late news on baff in autoimmune diseases. Autoimmun Rev 2010;9(12):804-806

33 Zhou Z, Chen Z, Li H, et al. BAFF and BAFF-R of peripheral blood and spleen mononuclear cells in idiopathic thrombocytopenic purpura. Autoimmunity 2009;42(02):112-119

34 Yang Q Xu S, Li X, et al. Pathway of toll-like receptor 7/B cell activating factor/B cell activating factor receptor plays a role in immune thrombocytopenia in vivo. PLoS One 2011;6(07):e22708

35 Yang $\mathrm{Q}$ Wang $\mathrm{B}, \mathrm{Yu} \mathrm{H}$, et al. TLR7 promotes Th1 polarization in immune thrombocytopenia. Thromb Res 2011;128(03):237-242

36 Li X, Zhong H, Bao W, et al. Defective regulatory B-cell compartment in patients with immune thrombocytopenia. Blood 2012; 120(16):3318-3325

37 Semple JW, Provan D. The immunopathogenesis of immune thrombocytopenia: $\mathrm{T}$ cells still take center-stage. Curr Opin Hematol 2012;19(05):357-362

38 Blair PA, Noreña LY, Flores-Borja F, et al. CD19(+)CD24(hi)CD38 (hi) B cells exhibit regulatory capacity in healthy individuals but are functionally impaired in systemic lupus erythematosus patients. Immunity 2010;32(01):129-140

39 Mauri C, Bosma A. Immune regulatory function of B cells. Annu Rev Immunol 2012;30:221-241

40 Chen JF, Yang LH, Dong CX, et al. Significance of detection of antiGPIIb/IIla antibody secreting B cells and platelet-specific antibody in patients with idiopathic thrombocytopenic purpura [in Chinese]. Zhonghua Xue Ye Xue Za Zhi 2010;31(09):603-606

41 Harrington WJ, Minnich V, Hollingsworth JW, Moore CV. Demonstration of a thrombocytopenic factor in the blood of patients with thrombocytopenic purpura. J Lab Clin Med 1951; 38(01):1-10

42 Stockelberg D, Hou M, Jacobsson S, Kutti J, Wadenvik H. Detection of platelet antibodies in chronic idiopathic thrombocytopenic purpura (ITP). A comparative study using flow cytometry, a whole platelet ELISA, and an antigen capture ELISA. Eur J Haematol 1996;56(1-2):72-77

43 He R, Reid DM, Jones CE, Shulman NR. Extracellular epitopes of platelet glycoprotein Ib alpha reactive with serum antibodies from patients with chronic idiopathic thrombocytopenic purpura. Blood 1995;86(10):3789-3796 
44 Kiefel V, Freitag E, Kroll H, Santoso S, Mueller-Eckhardt C. Platelet autoantibodies (IgG, IgM, IgA) against glycoproteins IIb/IIIa and $\mathrm{Ib} / \mathrm{IX}$ in patients with thrombocytopenia. Ann Hematol 1996;72 (04):280-285

45 McMillan R. The role of antiplatelet autoantibody assays in the diagnosis of immune thrombocytopenic purpura. Curr Hematol Rep 2005;4(02):160-165

46 McMillan R. The pathogenesis of chronic immune thrombocytopenic purpura. Semin Hematol 2007;44(04, Suppl 5):S3-S11

47 Tomiyama Y, Kosugi S. Autoantigenic epitopes on platelet glycoproteins. Int J Hematol 2005;81(02):100-105

48 Kiyomizu K, Kashiwagi H, Nakazawa T, et al. Recognition of highly restricted regions in the $\beta$-propeller domain of $\alpha$ IIb by platelet-associated anti- $\alpha$ IIb $\beta 3$ autoantibodies in primary immune thrombocytopenia. Blood 2012;120(07):1499-1509

49 van Leeuwen EF, van der Ven JT, Engelfriet CP, von dem Borne AE. Specificity of autoantibodies in autoimmune thrombocytopenia. Blood 1982;59(01):23-26

50 Fujisawa K, Tani P, O'Toole TE, Ginsberg MH, McMillan R. Different specificities of platelet-associated and plasma autoantibodies to platelet GPIIb-IIla in patients with chronic immune thrombocytopenic purpura. Blood 1992;79(06):1441-1446

51 Berchtold P, Wenger M. Autoantibodies against platelet glycoproteins in autoimmune thrombocytopenic purpura: their clinical significance and response to treatment. Blood 1993;81(05): $1246-1250$

52 McMillan R, Lopez-Dee J, Loftus JC. Autoantibodies to alpha(IIb) beta(3) in patients with chronic immune thrombocytopenic purpura bind primarily to epitopes on alpha(IIb). Blood 2001; 97(07):2171-2172

53 Roark JH, Bussel JB, Cines DB, Siegel DL. Genetic analysis of autoantibodies in idiopathic thrombocytopenic purpura reveals evidence of clonal expansion and somatic mutation. Blood 2002; 100(04):1388-1398

54 van Bladel ER, Laarhoven AG, van der Heijden LB, et al. Functional platelet defects in children with severe chronic ITP as tested with 2 novel assays applicable for low platelet counts. Blood 2014;123 (10):1556-1563

55 Frelinger AL III, Grace RF, Gerrits AJ, Carmichael SL, Forde EE, Michelson AD. Platelet function in ITP, independent of platelet count, is consistent over time and is associated with both current and subsequent bleeding severity. Thromb Haemost 2018;118 (01):143-151

56 Stasi R. Pathophysiology and therapeutic options in primary immune thrombocytopenia. Blood Transfus 2011;9(03): 262-273

57 Podolanczuk A, Lazarus AH, Crow AR, Grossbard E, Bussel JB. Of mice and men: an open-label pilot study for treatment of immune thrombocytopenic purpura by an inhibitor of Syk. Blood 2009;113(14):3154-3160

58 Asahi A, Nishimoto T, Okazaki Y, et al. Helicobacter pylori eradication shifts monocyte Fcgamma receptor balance toward inhibitory FcgammaRIIB in immune thrombocytopenic purpura patients. J Clin Invest 2008;118(08):2939-2949

59 Samuelsson A, Towers TL, Ravetch JV. Anti-inflammatory activity of IVIG mediated through the inhibitory Fc receptor. Science 2001;291(5503):484-486

60 Tsubakio T, Tani P, Curd JG, McMillan R. Complement activation in vitro by antiplatelet antibodies in chronic immune thrombocytopenic purpura. Br J Haematol 1986;63(02):293-300

61 Peerschke EI, Andemariam B, Yin W, Bussel JB. Complement activation on platelets correlates with a decrease in circulating immature platelets in patients with immune thrombocytopenic purpura. Br J Haematol 2010;148(04):638-645

62 Najaoui A, Bakchoul T, Stoy J, et al. Autoantibody-mediated complement activation on platelets is a common finding in patients with immune thrombocytopenic purpura (ITP). Eur J Haematol 2012;88(02):167-174
63 Bakchoul T, Greinacher A, Sachs UJ, et al. Inhibition of HPA-1a alloantibody-mediated platelet destruction by a deglycosylated anti-HPA-1a monoclonal antibody in mice: toward targeted treatment of fetal-alloimmune thrombocytopenia. Blood 2013; 122(03):321-327

64 Bakchoul T, Walek K, Krautwurst A, et al. Glycosylation of autoantibodies: insights into the mechanisms of immune thrombocytopenia. Thromb Haemost 2013;110(06):1259-1266

65 Segal JB, Powe NR. Prevalence of immune thrombocytopenia: analyses of administrative data. J Thromb Haemost 2006;4(11): 2377-2383

66 Nieswandt B, Bergmeier W, Rackebrandt K, Gessner JE, Zirngibl $\mathrm{H}$. Identification of critical antigen-specific mechanisms in the development of immune thrombocytopenic purpura in mice. Blood 2000;96(07):2520-2527

67 Webster ML, Sayeh E, Crow M, et al. Relative efficacy of intravenous immunoglobulin $G$ in ameliorating thrombocytopenia induced by antiplatelet GPIIbIIIa versus GPIbalpha antibodies. Blood 2006;108(03):943-946

$68 \mathrm{Li} \mathrm{J}$, van der Wal DE, Zhu G, et al. Desialylation is a mechanism of Fc-independent platelet clearance and a therapeutic target in immune thrombocytopenia. Nat Commun 2015;6:7737

69 Tao L, Zeng Q Li J, et al. Platelet desialylation correlates with efficacy of first-line therapies for immune thrombocytopenia. J Hematol Oncol 2017;10(01):46

70 Li J, Sullivan JA, Ni H. Pathophysiology of immune thrombocytopenia. Curr Opin Hematol 2018;25(05):373-381

71 Quach ME, Dragovich MA, Chen W, et al. Fc-independent immune thrombocytopenia via mechanomolecular signaling in platelets. Blood 2018;131(07):787-796

72 Mason KD, Carpinelli MR, Fletcher JI, et al. Programmed anuclear cell death delimits platelet life span. Cell 2007;128(06):1173-1186

$73 \mathrm{Li} \mathrm{C}$, Piran S, Chen P, et al. The maternal immune response to fetal platelet GPIb $\alpha$ causes frequent miscarriage in mice that can be prevented by intravenous IgG and anti-FcRn therapies. J Clin Invest 2011;121(11):4537-4547

74 van Der Wal DE, Zhu G, Li J, et al. Desialylation: a novel platelet clearance mechanism and a potential new therapeutic target in Anti-GPIb antibody mediated thrombocytopenia. Blood 2012; 120(21):265

75 Leytin V, Mykhaylov S, Starkey AF, et al. Intravenous immunoglobulin inhibits anti-glycoprotein IIb-induced platelet apoptosis in a murine model of immune thrombocytopenia. $\mathrm{Br} J$ Haematol 2006; 133(01):78-82

76 Alvarez Román MT, Fernández Bello I, Arias-Salgado EG, et al. Effects of thrombopoietin receptor agonists on procoagulant state in patients with immune thrombocytopenia. Thromb Haemost 2014;112(01):65-72

77 Winkler J, Kroiss S, Rand ML, et al. Platelet apoptosis in paediatric immune thrombocytopenia is ameliorated by intravenous immunoglobulin. Br J Haematol 2012;156(04):508-515

78 Goette NP, Glembotsky AC, Lev PR, et al. Platelet apoptosis in adult immune thrombocytopenia: insights into the mechanism of damage triggered by auto-antibodies. PLoS One 2016;11(08):e0160563

79 Grozovsky R, Begonja AJ, Liu K, et al. The Ashwell-Morell receptor regulates hepatic thrombopoietin production via JAK2-STAT3 signaling. Nat Med 2015;21(01):47-54

$80 \mathrm{Xu} \mathrm{M}$, Li J, Neves MAD, et al. GPIb $\alpha$ is required for plateletmediated hepatic thrombopoietin generation. Blood 2018;132 (06):622-634

81 Machlus KR, Italiano JE Jr. The incredible journey: from megakaryocyte development to platelet formation. J Cell Biol 2013; 201(06):785-796

82 Broudy VC, Lin NL, Kaushansky K. Thrombopoietin (c-mpl ligand) acts synergistically with erythropoietin, stem cell factor, and interleukin- 11 to enhance murine megakaryocyte colony growth and increases megakaryocyte ploidy in vitro. Blood 1995;85(07): $1719-1726$ 
83 Dameshek W, Miller EB. The megakaryocytes in idiopathic thrombocytopenic purpura, a form of hypersplenism. Blood 1946;1:27-50

84 Rolovic Z, Baldini M, Dameshek W. Megakaryocytopoiesis in experimentally induced immune thrombocytopenia. Blood 1970;35(02):173-188

85 McMillan R, Luiken GA, Levy R, Yelenosky R, Longmire RL. Antibody against megakaryocytes in idiopathic thrombocytopenic purpura. JAMA 1978;239(23):2460-2462

86 Takahashi R, Sekine N, Nakatake T. Influence of monoclonal antiplatelet glycoprotein antibodies on in vitro human megakaryocyte colony formation and proplatelet formation. Blood 1999;93(06):1951-1958

87 Chang M, Nakagawa PA, Williams SA, et al. Immune thrombocytopenic purpura (ITP) plasma and purified ITP monoclonal autoantibodies inhibit megakaryocytopoiesis in vitro. Blood 2003;102(03):887-895

88 McMillan R, Wang L, Tomer A, Nichol J, Pistillo J. Suppression of in vitro megakaryocyte production by antiplatelet autoantibodies from adult patients with chronic ITP. Blood 2004;103(04): 1364-1369

89 Iraqi M, Perdomo J, Yan F, Choi PY, Chong BH. Immune thrombocytopenia: antiplatelet autoantibodies inhibit proplatelet formation by megakaryocytes and impair platelet production in vitro. Haematologica 2015;100(05):623-632

90 Zeng DF, Chen F, Wang S, et al. Autoantibody against integrin $\alpha_{\mathrm{v}}$ $\beta_{3}$ contributes to thrombocytopenia by blocking the migration and adhesion of megakaryocytes. J Thromb Haemost 2018;16 (09):1843-1856

91 Kuwana M, Okazaki Y, Kajihara M, et al. Autoantibody to c-Mpl (thrombopoietin receptor) in systemic lupus erythematosus: relationship to thrombocytopenia with megakaryocytic hypoplasia. Arthritis Rheum 2002;46(08):2148-2159

92 Kuter DJ. Biology and chemistry of thrombopoietic agents. Semin Hematol 2010;47(03):243-248

93 Wang B, Nichol JL, Sullivan JT. Pharmacodynamics and pharmacokinetics of AMG 531, a novel thrombopoietin receptor ligand. Clin Pharmacol Ther 2004;76(06):628-638

94 Mahévas M, Fain O, Ebbo M, et al. The temporary use of thrombopoietin-receptor agonists may induce a prolonged remission in adult chronic immune thrombocytopenia. Results of a French observational study. Br J Haematol 2014;165(06): 865-869

95 Saleh MN, Bussel JB, Cheng G, et al; EXTEND Study Group. Safety and efficacy of eltrombopag for treatment of chronic immune thrombocytopenia: results of the long-term, open-label EXTEND study. Blood 2013;121(03):537-545

96 Bal G, Fabian D, Maia D, Ringel F, Salama A. Effect of thrombopoietin receptor agonists on leukocyte and haematopoietic stem and progenitor cells in the peripheral blood of patients with immune thrombocytopenic purpura. Ann Hematol 2017;96(12): 2045-2056

97 Sheng GY, Huang XL, Bai ST. Expression levels of CXCR4 on megakaryocytes and its ligand in bone marrow in children with acute idiopathic thrombocytopenic purpura [in Chinese]. Zhonghua Er Ke Za Zhi 2004;42(07):499-501

98 Edelstein LC, Bray PF. MicroRNAs in platelet production and activation. Blood 2011;117(20):5289-5296

99 Garzon R, Pichiorri F, Palumbo T, et al. MicroRNA fingerprints during human megakaryocytopoiesis. Proc Natl Acad Sci U S A 2006;103(13):5078-5083
100 Bay A, Coskun E, Oztuzcu S, Ergun S, Yilmaz F, Aktekin E. Plasma microRNA profiling of pediatric patients with immune thrombocytopenic purpura. Blood Coagul Fibrinolysis 2014;25(04): 379-383

101 Basciano PA, Bussel J, Hafeez Z, Christos PJ, Giannakakou P. The beta 1 tubulin $\mathrm{R} 307 \mathrm{H}$ single nucleotide polymorphism is associated with treatment failures in immune thrombocytopenia (ITP). Br J Haematol 2013;160(02):237-243

102 Yang L, Wang L, Zhao CH, et al. Contributions of TRAIL-mediated megakaryocyte apoptosis to impaired megakaryocyte and platelet production in immune thrombocytopenia. Blood 2010;116 (20):4307-4316

103 Radley JM, Haller CJ. Fate of senescent megakaryocytes in the bone marrow. Br J Haematol 1983;53(02):277-287

104 Houwerzijl EJ, Blom NR, van der Want JJ, et al. Ultrastructural study shows morphologic features of apoptosis and para-apoptosis in megakaryocytes from patients with idiopathic thrombocytopenic purpura. Blood 2004;103(02):500-506

105 Arnold DM, Nazi I, Kelton JG. New treatments for idiopathic thrombocytopenic purpura: rethinking old hypotheses. Expert Opin Investig Drugs 2009;18(06):805-819

106 Vrbensky JR, Nazy I, Toltl LJ, et al. Megakaryocyte apoptosis in immune thrombocytopenia. Platelets 2018;29(07):729-732

107 Guo L, Kapur R, Aslam R, et al. Antiplatelet antibody-induced thrombocytopenia does not correlate with megakaryocyte abnormalities in murine immune thrombocytopenia. Scand J Immunol 2018;88(01):e12678

$108 \mathrm{Kim} \mathrm{N}$, Im KI, Lim JY, et al. Mesenchymal stem cells for the treatment and prevention of graft-versus-host disease: experiments and practice. Ann Hematol 2013;92(10):1295-1308

109 Xiao J, Zhang C, Zhang Y, et al. Transplantation of adipose-derived mesenchymal stem cells into a murine model of passive chronic immune thrombocytopenia. Transfusion 2012;52(12):2551-2558

110 Madonna R, Petrov L, Teberino MA, et al. Transplantation of adipose tissue mesenchymal cells conjugated with VEGF-releasing microcarriers promotes repair in murine myocardial infarction. Cardiovasc Res 2015;108(01):39-49

111 Mohanty ST, Kottam L, Gambardella A, et al. Alterations in the selfrenewal and differentiation ability of bone marrow mesenchymal stem cells in a mouse model of rheumatoid arthritis. Arthritis Res Ther 2010;12(04):R149

112 Li X, Liu L, Meng D, et al. Enhanced apoptosis and senescence of bonemarrow-derived mesenchymal stem cells in patients with systemic lupus erythematosus. Stem Cells Dev 2012;21(13):2387-2394

113 Bacigalupo A, Valle $M$, Podestà $M$, et al. T-cell suppression mediated by mesenchymal stem cells is deficient in patients with severe aplastic anemia. Exp Hematol 2005;33(07):819-827

114 Zhang D, Li H, Ma L, et al. The defective bone marrow-derived mesenchymal stem cells in patients with chronic immune thrombocytopenia. Autoimmunity 2014;47(08):519-529

115 Pérez-Simón JA, Tabera S, Sarasquete ME, et al. Mesenchymal stem cells are functionally abnormal in patients with immune thrombocytopenic purpura. Cytotherapy 2009;11(06):698-705

116 Tao Y, Song D, Zhang F, Ren S, Zhang H, Sun L. Transplantation of bone-marrow-derived mesenchymal stem cells into a murine model of immune thrombocytopenia. Blood Coagul Fibrinolysis 2017;28(08):596-601

117 Wang X, Yin X, Sun W, et al. Intravenous infusion umbilical cordderived mesenchymal stem cell in primary immune thrombocytopenia: a two-year follow-up. Exp Ther Med 2017;13(05): $2255-2258$ 